\section{$\underset{\text { hommes }}{\text { \& migrations }}$}

\section{Hommes \& migrations}

Revue française de référence sur les dynamiques

migratoires

$1300 \mid 2012$

Nouveaux modèles migratoires en Méditerranée

\title{
Cai luong
}

\section{(2) OpenEdition \\ Journals}

Édition électronique

URL : http://journals.openedition.org/hommesmigrations/947

DOI : 10.4000/hommesmigrations.947

ISSN : 2262-3353

Éditeur

Musée national de l'histoire de l'immigration

Édition imprimée

Date de publication : 1 novembre 2012

Pagination : 154-157

ISSN : 1142-852X

\section{Référence électronique}

"Cai luong », Hommes \& migrations [En ligne], 1300 | 2012, mis en ligne le 29 mai 2013, consulté le 22 septembre 2020. URL : http://journals.openedition.org/hommesmigrations/947 ; DOI : https://doi.org/ 10.4000/hommesmigrations.947

Ce document a été généré automatiquement le 22 septembre 2020.

Tous droits réservés 


\section{Cai luong}

\section{NOTE DE L'AUTEUR}

Spectacle Da Co - Tambour dans la nuit 8 et 9 mars 2013, auditorium du musée Guimet.

1 Huong Thanh a grandi dans l'ombre d'un père artiste, comédien et chanteur adulé : Huu Phuoc, vedette du cai luong. À Saigon, sa maison vivait au rythme des musiciens qui venaient y répéter. Les coulisses du théâtre où il jouait chaque soir n'avaient pas de secret pour sa fille. Les messieurs s'y transformaient en rois caressant leur longue barbe. Les dames y devenaient de merveilleuses princesses drapées de soies multicolores, dans lesquelles scintillaient or et diamants, bijoux et perles fines. Petite fille, Huong Thanh appartient à cet univers de fantaisie qu'elle adore jusqu'à vouloir en faire son métier. Las, elle n'est que la cadette et c'est sa sœur aînée qui a bénéficié, dès l'âge de six ans, de l'initiation de son père. Qu'à cela ne tienne, elle est tellement imprégnée de l'univers qui l'entoure, assistant aux répétitions, s'exerçant à chanter pour elle-même, qu'elle révèle à son père l'ampleur de ses dispositions. Convaincu de son talent, celui-ci la recommande à de bons professeurs. À 10 ans, Huong Thanh entre ainsi dans le monde du théâtre chanté dont elle a si longtemps rêvé. Sept ans plus tard, elle intègre une troupe professionnelle. Mais le rêve est de courte durée. Après la fin de la guerre, le cai luong ne fait plus recette à Hô Chi Minh-Ville. Huu Phuoc décide de s'exiler en France avec ses enfants. Chaque année la magie du cai luong rejaillit à l'occasion des fêtes vietnamiennes, accentuant un peu la nostalgie de la vie d'autrefois. Et dès lors, avec ténacité, Huong Thanh œuvre à faire découvrir la beauté de ce genre en déclin depuis le milieu des années 1970.

\section{La musique vietnamienne et le cai luong}

2 Lauréate du prix France Musique des musiques du monde en 2007, la chanteuse consacre au cai luong l'album qui la récompense. C'est l'occasion de renouer avec son pays d'origine en y réalisant son rêve d'enregistrer à Saigon, où elle interprète plusieurs airs de cai luong en duo avec sa sœur aînée Huong Lan, devenue vedette de la 
chanson vietnamienne. "Huong Thanh, née d'une famille d'acteurs chevronnés du cai luong, a reçu de son père une éducation artistique exceptionnelle. Malgré son long séjour en France, elle a su conserver la belle et authentique tradition de cet art théâtral spécifique du sud du Vietnam. Belle voix, ornementation originale des mélodies, strict respect des hoi et dieu (nuance modale, système modal). Ses prestations donnent un exemple du hat cai luong beau et authentique", écrit l'éminent professeur Trân Van Khê en exergue de ce disque publié par Ocora Radio France.

En 2012, à l'occasion d'un nouveau voyage au Vietnam, Huong Thanh décide d'inviter l'une des dernières détentrices du grand art du cai luong à se produire sur scène à ses côtés en France. La chanteuse et actrice Ngoc Giau maîtrise tous les secrets de jeu qui florissaient à l'époque où les théâtres de Saigon étaient pleins d'un public populaire passionné. Ayant interprété plus d'une centaine de rôles, tant féminins que masculins, au cours d'une carrière débutée à l'âge de 13 ans, elle a été nommée Artiste du Peuple, plus haut titre de reconnaissance décerné par l'État vietnamien pour la qualité de son art. Ainsi se démarque-t-elle des jeunes vedettes du cai luong contemporain qui, plutôt que de jouer devant un public, enregistrent seulement pour la télévision ou pour des vidéos revendues bon marché. Huong Thanh a résolu de présenter en France la qualité d'un art flamboyant tel qu'il se jouait sur les scènes des théâtres. Elle sera guidée par son aînée Ngoc Giau à qui elle donnera la réplique dans les extraits de deux pièces majeures du répertoire de cai luong: Adieu de la concubine Yuji à Xiang Yu, pièce historique de Bach Mai, et L'Attente du retour de l'enfant, une pièce contemporaine de Vien Chau. Deux musiciens traditionnels chevronnés, venus tout spécialement du Vietnam, accompagneront les deux chanteuses : le multi-instrumentiste et compositeur Thai An et le percussionniste et professeur au Conservatoire nationale de Hanoi Quôc Hung. Il faut ici saluer la détermination de la chanteuse Huong Thanh, qui a pris l'initiative de ce projet inédit, d'une grande valeur culturelle.

\section{Les structures essentielles de la musique vietnamienne}

L'approche du cai luong nécessite de connaître certaines règles à la base de la musique vietnamienne. Le professeur Trân Van Khê, exilé pendant de nombreuses années en France, où il a publié de nombreux ouvrages de référence sur les musiques d'Asie et enregistré des disques lumineux de musique vietnamienne, vit aujourd'hui à Hô Chi Minh-Ville. Sa maison, qui lui a été offerte par le gouvernement vietnamien, renferme l'ensemble des collections de livres, partitions, instruments et phonogrammes qu'il a pu rassembler au cours de sa longue carrière de musicien et de maître en musicologie. Elle sera consacrée à son souvenir et à son œuvre "après son grand départ".

5 Professeur Trân Van Khê: "Un décret français du XIXe siècle a fixé le la de la musique occidentale à 435 ou 440 vibrations doubles par seconde dans une température de $16^{\circ}$ ou $20^{\circ}$ centigrades sous une pression atmosphérique de $76 \mathrm{~mm}$ de mercure. Mais au Vietnam, il n'y a pas de hauteur absolue. Chaque artiste peut avoir son 'ho'. La musique vietnamienne n'est pas constituée de notes, mais de cinq degrés: 'ho' est le premier degré, celui de la fondamentale, 'xu' est la seconde majeure au-dessus de 'ho', 'xang', la quarte, 'xê', la quinte, 'công', la sixte, 'liu', l'octave. Mais ces notes ne sont pas fixes: elles peuvent bouger. Lorsque la fréquence varie vers le grave, on l'appelle 'non' (c'est-à-dire 'jeune'), et vers l'aigu, on l'appelle 'già' (c'est-à-dire 
'vieux'). Ces variations ne correspondent pas précisément au bémol ou au dièse, elles dépendent du jugement de l'artiste et de celui du public.

Il existe une marge de tolérance à l'intérieur de laquelle toutes les notes sont valables, mais audelà de laquelle l'interprète se trouve dans l'erreur. Tout dépend de la manière de chanter la succession des notes. C'est pourquoi la musique vietnamienne est établie selon une conception de structures dynamiques et ouvertes, à la différence de la musique classique occidentale, dont la structure statique est fermée. Fermée, parce que, lorsqu'une pièce est créée, on ne peut plus la changer. Le musicien doit suivre exactement la partition écrite, et ne sont tolérées que certaines nuances d'exécution. Au Vietnam, non seulement l'interprète a la possibilité de changer, mais il doit le faire s'il a atteint un certain niveau. Il se réfère à la règle dite: 'chan (vrai), phuong (carré), hoa (fleur) la (feuille)'. L'interprète doit connaître par cœur le schéma de la pièce, auquel il ne peut pas toucher. Il doit jouer 'vrai et carré'. Mais il doit également savoir introduire des variations dans le cadre de cette structure fixe, c'est-à-dire ajouter 'les fleurs et les feuilles'. Ce principe est valable pour la mélodie comme pour le rythme. Dans le cai luong, l'art de l'artiste consiste précisément à savoir ajouter 'les fleurs et les feuilles'. Produire une mélodie presque nouvelle, trouver un style d'exécution nouveau, voilà ce qui détermine le caractère et l'originalité de l'artiste."

\section{La naissance de l'art du cai luong}

Professeur Trân Van Khê : "Littéralement, cai luong veut dire 'changer pour embellir' ('cai' : changer, 'luong': plus beau). Il s'agissait de changer l'organisation de la scène, de changer l'habillement des personnages, mais aussi le placement de la voix. Jusqu'alors au théâtre, on employait la voix de fausset, la voix pharyngienne, la voix de poitrine, du foie, des entrailles. Avec le cai luong l'artiste doit utiliser la voix ordinaire, mais bien posée. Ce genre a servi en quelque sorte de champ d'expérimentation pour la transformation du théâtre chanté. À l'origine, le hat cai luong dérive du 'ca-ra-bo', qui consistait à 'chanter en faisant des gestes'. Le répertoire du ca-ra-bo venait de la musique de chambre 'dan tai tu', musique des artistes du Sud qui accompagnaient leur chant de gestes très simples. Par la suite, ces gestes sont devenus plus stylisés, plus artistiques, plus codifiés. Le cai luong est né de la recherche d'un style nouveau et s'est développé en plusieurs étapes. De 1920 à 1923, il s'agit du style traditionnel. Entre 1923 et 1925, il s'inspire du style chinois qui était joué dans le Sud et qui plaisait beaucoup. L'apogée de cette période se situe en 1925 avec la troupe de Phu Kung, qui a utilisé dans ses spectacles les gestes $d u$ hat tuong (théâtre du Nord), du hat boi (théâtre du Sud), ainsi que les danses du théâtre chinois. En 1927 apparaissent deux courants: l'un empruntant au théâtre chinois, l'autre plutôt tourné vers la tradition vietnamienne. Diverses tendances sont apparues ensuite: bouddhique, religieuse, universel merveilleux, etc. L'un des grands acteurs du cai luong, Nam Chau, a dit qu'il fallait jouer 'vrai, mais d'une belle manière'. Dans le théâtre traditionnel, lorsque l'on boit, il faut ouvrir l'éventail, prendre la tasse, la cacher derrière l'éventail, boire, jeter le reste par-dessus l'épaule gauche, reposer la tasse et caresser sa barbe. À la question de savoir pourquoi l'acteur du théâtre vietnamien ne boit pas le thé comme tout le monde, le maître répond: 'Si vous voulez voir quelqu'un qui boit comme tout le monde, allez chez le voisin! Mais si vous voulez voir quelqu'un qui boit avec les gestes stylisés de la beauté, de la sérénité et du caractère artistique, allez au théâtre !' Ainsi, dans le cai luong, l'acteur se doit de jouer vrai, mais de belle manière." 


\section{Le vong cô}

8 L'air Da cô Hoai Lang a donné naissance à un genre spécifique au cai luong, sous le nom de "vong cô", c'est-à-dire "regret du passé". Le public attend impatiemment le moment du vong cô, incontournable dans le développement dramatique d'une pièce. Dans son ouvrage Vietnam, les traditions musicales (Buchet-Chastel, 1967), le professeur Trân Van Khê nous enseigne que "plusieurs acteurs au talent médiocre gagnent largement leur vie uniquement parce qu'ils chantent remarquablement le vong cô". Cette assertion, sans doute bien vraie lorsqu'il la formula en 1967, n'est plus vraiment d'actualité. Dans La Musique vietnamienne traditionnelle (Presse universitaire de France, 1962), le professeur fournit l'histoire de l'apparition de cet air nouveau : "Vers 1919, le 'Vong cô Hoai Lang' fut écrit par Ong Sâu Lâu, d'après le témoignage rapporté par le journaliste vietnamien Thanh Cao, quand le musicien fut obligé de divorcer d'avec sa femme, après huit ans de vie commune. En souvenir de cet amour, il écrivit Hoai Lang qui, dans la pensée du musicien, signifiait 'en pensant à la bien-aimée."

Lorsqu'il joue cet air triste devant un metteur en scène, celui-ci l'enjoint à en changer le titre en Vong cô (le regret du passé). Le musicien additionnera les deux titres. Quant au vong cô, il deviendra un genre à part entière. Écrit à l'origine en 20 phrases de 2 mesures, cette pièce est adoptée par toutes les troupes de théâtre au Sud. "Les phrases de 2 mesures étant trop courtes pour exprimer une tristesse profonde, les musiciens du sud du Vietnam les ont transformées en phrases de 4 mesures, poursuit le professeur. Par la suite, le vong cô n'a cessé d'évoluer dans ce sens." Les phrases passent de 8 à 16, puis à 32 mesures. "Le vong cô devient ainsi trop long et dans les réunions musicales ou sur les scènes du théâtre rénové, on n'en joue que les 6 premières phrases", précise le professeur Trân Van Khê.

\section{Le cai luong aujourd'hui}

10 Professeur Trân Van Khê : "Le hat cai luong a été en quelque sorte tué par le karaoké et la vidéo. La vidéo aurait pu être un collaborateur extraordinaire pour le cai luong, en aidant cet art théâtral à se propager dans l'espace et dans le temps. Or, en présentant des pièces nouvelles, interprétées par de grands artistes payés très cher pour figurer sur ces vidéos inédites, la vidéo a coupé l'herbe sous le pied du cai luong. C'est sur le petit écran qu'aujourd'hui le public découvre les nouvelles pièces et non pas au théâtre. Certes, il est plus avantageux de se procurer une vidéo que l'on peut regarder en famille et faire circuler auprès de ses amis, que d'aller au théâtre. Mais ce que l'on oublie, c'est que les artistes qui jouent pour la vidéo ne sont pas en face d'un public qui répond et avec lequel se tisse un lien invisible. Ils jouent pour une machine. Lorsque le public voit les acteurs mis en boite, la chaleur disparaît. Au lieu de sentir l'essence de l'art, il n'en voit que l'apparence.

11 Aujourd'hui, le cai luong manque d'auteurs, de directeurs de théâtre pour produire es spectacles, de bonnes troupes et de public. Il y a quelque temps, on aurait pu penser que cet art serait appelé à tomber en désuétude, même dans l'oubli. Or on assiste actuellement à une sorte de renaissance. Une nouvelle politique du gouvernement semble se dessiner pour aider le cai luong à redémarrer. Mais c'est une aide politique, pas une aide concrète. Les artistes de cai luong rencontrent des difficultés pour vivre et ne bénéficient pas d'une grande considération. C'est pourquoi je me suis beaucoup investi pour prendre la défense du cai luong et affirmer, à travers mes écrits, qu'il ne s'agit pas d'un mode de distraction, mais d'un art à part entière." 


\section{RÉSUMÉS}

Huong Thanh déploie l'art du cai luong, théâtre chanté vietnamien, pour deux soirées exceptionnelles au musée Guimet à Paris les 8 et 9 mars 2013. Ce bel hommage rendu par la chanteuse à feu son père Huu Phuoc, star du genre à Saigon, est aussi une façon d'offrir au cai luong, délaissé au Vietnam, une reconnaissance méritée. 